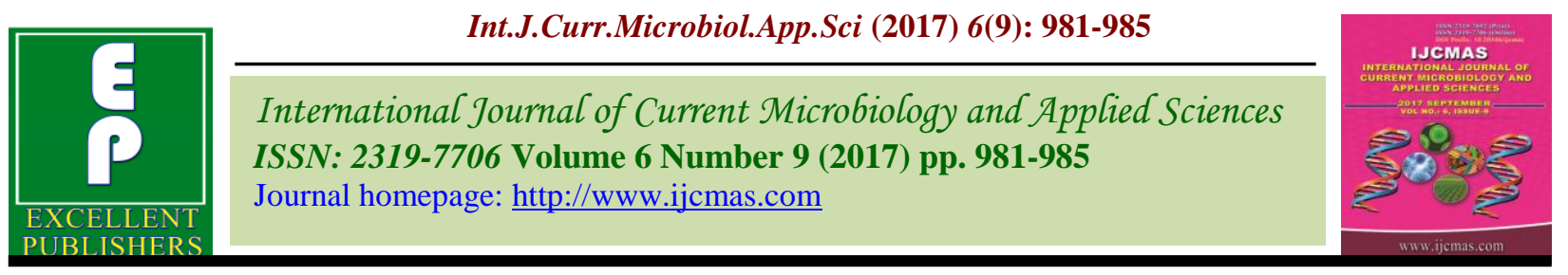

Original Research Article

https://doi.org/10.20546/ijcmas.2017.609.119

\title{
Bacteriological Study of Acute Otitis Externa in a Tertiary Care Hospital of a District in North Karnataka, India
}

\author{
Shilpa K. Gokale, Anushka Devinkar*, Drsuresh Sonth and S.S. Solabannavar \\ Department of microbiology, S N Medical College, Navanagar, \\ Bagalkot, Karnataka 587103, India \\ *Corresponding author
}

\section{A B S T R A C T}

\section{Keywords}

Otitis externa, Bacterial infection, Antibiotics.

Article Info

Accepted:

17 July 2017

Available Online:

10 September 2017
Otitis externa is an inflammation or infection of the outer ear canal. It is usually associated with secondary bacterial and/or fungal infection of macerated skin and subcutaneous cellular tissue. Aim of the study is to isolate and identify species of bacteria in patients with clinical diagnosis of Acute Otitis Externa and to know the susceptibility of microorganisms to the antimicrobial drugs. This study involved 103 patients with otitis externa. Two sets of samples were collected from their ears, one set was used for slide preparations, and the other for microbial culturing. After culturing, the microorganisms were identified by conventional methods. $\mathrm{S}$ aureus was the most common organism isolated followed by Klebsiella pneumoniae, and coagulase negative staphylococcus. Aminoglycosides and Quinolones showed variable activity against all bacteria tested and small percentage of bacteria were resistant to Carbapenem drugs. Acute Otitis Externa is a polymicrobial infection and knowledge regarding micro-organisms etiology and susceptibility will contribute to rational antibiotic usage and treatment success.

\section{Introduction}

Otitis externa is an inflammation of the outer ear. It includes the inflammatory conditions of the auricle, external auditory canal and outer surface of the eardrum $(1,2)$. It can be localized or diffuse and may be acute or chronic. It is a common ear condition across individuals of all ages. Acute otitis externa is a polymicrobial infectious disease $(3,4,5)$. Frequently associated pathogens include $\mathrm{P}$ aeruginosa, S. epidermidis and S. aureus.

The most important environmental factor is excessive moisture. Waste materials absorb water and provide a suitable medium for bacterial growth $(6,7)$. Systemic conditions such as anemia, low vitamin concentration in the body, diabetes and dermatitis such as seborrhea, psoriasis and eczema may reduce resistance to infection in external ear, causing development of otitis externa $(8,9)$.

Despite the fact that our climatic condition may encourage Otitis externa, literature search reveals that not much work has been carried out in our district.

Keeping in view the high prevalence of otitis externa in hot, humid and dusty areas, following study was done. The aim of the study was to isolate and identify species of bacteria in patients with clinical diagnosis of acute otitis externa and to know the 
susceptibility of microorganisms to the antimicrobial drugs.

\section{Materials and Methods}

Study group: The samples were obtained from 103 patients attending the Otorhinolaryngology clinic of HSK hospital Bagalkot between July 2011 and June 2012 with the clinical diagnosis of otitis externa.

Collection of sample: ENT specialists obtained pairs of ear specimens using a sterile cotton swab from the external auditory canal

Bacteriological investigation: A set of specimens were stained by gram staining procedure and were microscopically studied.

Another set was inoculated on to chocolate agar and MacConkey agar. The inoculated media were incubated at $37^{\circ} \mathrm{c}$ for $18-24 \mathrm{hrs}$. Bacterial colonies were identified according to the standard bacteriological methods.

All the isolates were subjected to antibiotic sensitivity testing by Kirby Bauer disk diffusion method. Testing was specifically done for ciprofloxacin, gentamicin and chloramphenicol, which were available locally as topical ear drops.

\section{Results and Discussion}

A total of 103 patients with otitis externa were included in the study. The common symptoms presenting solely or in combination of each other encountered in the study group have been summarized in table 1. Pain (91\%) was the most common complaint encountered followed by ear discharge (51\%), itching $(39.8 \%)$, hearing loss $(19.4 \%)$ and fullness of ear $(5.8 \%)$.

There were 58 males and 45 females in our study. The age ranged from 2-70 years (Table 2). The most prevalent age group was $30-39$
(31), followed by $20-29$ (26) and $40-49$ (15).

In the study group, 85 samples were positive for the presence of organisms in gram's stain, of which 82 were both microscopy and culture positive, whereas 6 samples were positive only by culture and 3 samples were positive only by microscopy.

A total of 88 out of 103 samples were positive for bacterial growth by culture. The most common bacterial isolate was $S$. aureus 26 (46\%), followed by $K$. pneumoniae 15 (26.7\%), CONS 9 (10.7\%). By the disc diffusion method, Staphylococcus aureus showed $0 \%$ sensitivity to ampicillin followed by $26.9 \%$ to erythromycin, $30 \%$ ciprofloxacin and maximum sensitivity $92.3 \%$ gentamicin. All CONS isolates showed good sensitivity to gentamicin, erythromycin and clindamycin (Table 3-5).

One hundred and three patients with otitis externa were enrolled after examination. Among these patients, $56.3 \%$ were males and the rest were females. Battikhi et al., studied 180 patients, of these $55.5 \%$ were males (10), Cheong et al., studied 91 patients and found a frequency of $52.7 \%$ of male patients (11). Burgos et al., reported that $56 \%$ of patients were males (12). Our results correlate with most of the studies.

In our study middle aged individuals were more often affected than the other groups. Rowlands et al., reported it to be common in all the age groups (13). These results were not in agreement with our findings, this may be due to differences in life style, temperature and access to health care centers among the different population.

Pain and discharge were the most common symptom followed by itching hearing loss and fullness of ear, confirming the finding of earlier studies. 
Table.1 Symptomatology of otitis externa

\begin{tabular}{|l|l|}
\hline Symptoms & Incidence \\
\hline Pain & 94 \\
\hline Discharge & 53 \\
\hline Itching & 41 \\
\hline Hearing loss & 20 \\
\hline Fullness of ear & 06 \\
\hline
\end{tabular}

Table.2 Age and sex distribution of otitis externa patients

\begin{tabular}{|l|l|l|l|}
\hline Age in years & Male & Female & Total \\
\hline $0-19$ & 5 & 6 & 11 \\
\hline $20-29$ & 15 & 11 & 26 \\
\hline $30-39$ & 16 & 15 & 31 \\
\hline $40-49$ & 13 & 02 & 15 \\
\hline 50 \& above & 09 & 11 & 20 \\
\hline Total & 58 & 45 & 103 \\
\hline
\end{tabular}

Table. 3 Spectrum of bacterial isolates $n=88$

\begin{tabular}{|l|c|c|}
\hline Isolate & No of samples & Percentage \\
\hline S. aureus & 41 & 46 \\
\hline K. pneumoniae & 23 & 26.1 \\
\hline CONS & 14 & 15.9 \\
\hline Pseudomonas & 10 & 11.3 \\
\hline
\end{tabular}

Table.4 Antibiogram of S. aureus and CONS

\begin{tabular}{|l|c|c|c|c|}
\hline \multirow{2}{*}{} & \multicolumn{2}{|c|}{ S. aureus $\mathrm{n}=41$} & \multicolumn{2}{c|}{ CONS n=14 } \\
\cline { 2 - 5 } & No & $\%$ sensitive & No & $\%$ sensitive \\
\hline Ampicillin & 0 & 0 & 0 & 0 \\
\hline Erythromycin & 11 & 26.8 & 09 & 64.2 \\
\hline Clindamycin & 11 & 26.8 & 09 & 64.2 \\
\hline Ciprofloxacin & 12 & 29.2 & 09 & 64.2 \\
\hline Cephalexin & 28 & 68.2 & 14 & 100 \\
\hline Gentamycin & 38 & 92.6 & 14 & 100 \\
\hline
\end{tabular}

Table.5 Antibiogram of Klebsiella $\mathrm{n}=23$

\begin{tabular}{|l|l|l|}
\hline & No & $\%$ sensitive \\
\hline Ciprofloxacin & 18 & 78.2 \\
\hline Ceftazidime & 20 & 86.9 \\
\hline Piperacillin tazobactam & 20 & 86.9 \\
\hline Gentamycin & 21 & 91.3 \\
\hline Ceftriaxone & 21 & 91.3 \\
\hline Imipenem & 23 & 100 \\
\hline
\end{tabular}


Table.6 Antibiogram of Pseudomonas $\mathrm{n}=10$

\begin{tabular}{|l|l|l|}
\hline & No & $\%$ sensitive \\
\hline Chloramphenicol & 05 & 50 \\
\hline Ceftazidime & 07 & 70 \\
\hline Piperacillin tazobactam & 07 & 70 \\
\hline Ciprofloxacin & 08 & 80 \\
\hline Gentamycin & 08 & 80 \\
\hline Imipenem & 10 & 100 \\
\hline
\end{tabular}

In our study 88 patients were proven to be suffering with otitis externa by culture growth. It accounts to $85 \%$ of patients suspected to be having otitis externa were confirmed in our study. It is in accordance with other studies. In this study 82 samples were microscopy and culture positive, 6 samples were positive by culture and negative by microscopy. 3 samples did not show growth on media, although was positive in direct microscopy, probably because of delay in processing of these samples or may be the patient was already on antibiotics.

Culture results of specimens in media showed that the most common bacterium involved was $S$. aureus, followed by Klebsiella and CONS. Roland et al., reported $P$. aeruginosa, $S$. epidermidis, and $S$. aureus were the common isolates (14). Al Asaf et al., reported $P$. aeruginosa (39\%) and $S$. aureus $(18 \%)$ were the most common isolates (15). Nougueria et al., isolated $S$. aureus as the most common isolate in their study (16). Thus in most studies $P$. aeruginosa and $S$. aureus were reported as common isolates, which is consistent with our result.

Staphylococcus aureus was sensitive to gentamicin $(92.6 \%)$, cephalexin $(68.2 \%)$ and ciprofloxacin (29.2\%). Staphylococcus sensitivity to ciprofloxacin is in agreement with other studies. Most of the investigators reported high sensitivity rate for gentamicin.
Klebsiella showed high sensitivity to gentamicin, ciprofloxacin and ceftazidime.

Pseudomonas isolates showed high sensitivity to imipenem (100\%), ciprofloxacin $(83 \%)$, gentamicin $(83 \%)$ and piperacillin (66\%). High fluoroquinolones antibacterial activity against Pseudomonas was reported by others, although resistant strains of Pseudomonas to fluoroquinolones were detected in other studies (Tables 4-6).

In this study special interest was taken in to account of the common antibiotics prescribed by the ENT surgeons in treating ear discharge namely chloramphenicol, gentamicin and ciprofloxacin. Our study also correlates with other studies which show that the quinolone ciprofloxacin to be safe and effective particularly against $P$. aeruginosa and $S$. aureus.

Our present study, in the light of other studies, indicates that there can be a variation in the organisms infecting and their susceptibility pattern. Continuous and periodic evaluation of microbial pattern and antibiotic sensitivity of otitis externa helps to decrease the potential risk of complications.

\section{References}

1. Roland PS, Marple BF. Disorders of the external auditory canal. $J \mathrm{Am}$ AcadAudiol. 1997;8(6):367-78.

2. Ostrowski VB, Wiet RJ. Pathologic conditions of the external ear and 
auditory canal. Postgrad Med. 1996;100(3):223-8.

3. Hui CP, Canadian Paediatric Society ID, Immunization C. Acute otitis externa. Paediatr Child Health. 2013;18(2):96-101.

4. Beers SL, Abramo TJ. Otitis externa review. PediatrEmerg Care. 2004;20(4):250-6.

5. Kuczkowski J, Samet A, Brzoznowski W. [Bacteriologic evaluation of otitis externa and chronic otitis media]. Otolaryngol Pol. 2000;54(5):551-6.

6. Sander R. Otitis externa: a practical guide to treatment and prevention. $A m$ Fam Physician. 2001;63(5):927-36.

7. Osguthorpe JD, Nielsen DR. Otitis externa: Review and clinical update. Am Fam Physician. 2006;74(9):15106.

8. Mosges R, Nematian-Samani M, Eichel A. Treatment of acute otitis externa with ciprofloxacin otic $0.2 \%$ antibiotic ear solution. TherClin Risk Manag. 2011;7:325-36. doi: 10.2147/TCRM.S6769.

9. Mittal A, Kumar S. Role of $\mathrm{pH}$ of External Auditory Canal in Acute Otitis Externa. Indian J Otolaryngol Head Neck Surg. 2014;66(1):86-91. doi: 10.1007/s12070-013-0684-0.

10. Battikhi MN, Ammar SI. Otitis externa infection in Jordan. Clinical and microbiological features. Saudi Med J. 2004;25(9):1199-203.
11. Cheong CS, Tan LM, Ngo RY. Clinical audit of the microbiology of otorrhoea referred to a tertiary hospital in Singapore. Singapore Med J. 2012;53(4):244-8.

12. Burgos Sanchez A, MenachesGuardiola MI, Gras Albert JR, Talavera Sanchez J. KiakojuriK, Omran SM, Jalili B, Hajiahmadi M, Bagheri M, Shahandashti EF and RajabniaR. Bacterial otitis externa in patients attending an ENT clinic in Babol, North of Iran. Jundishapur $\mathbf{J}$ Microbiol. 2016 February; 9 (2): e23093

13. Rowlands S, Devalia H, Smith C, Hubbard R, Dean A. Otitis externa in UK general practice: a survey using the UK General Practice Research Database. $\mathrm{Br} \quad \mathrm{J}$ Gen Pract. 2001;51(468):533-8.

14. Roland PS, Stroman DW. Microbiology of acute otitis externa. Laryngoscope. 2002;112(7 Pt 1):116677. doi: 10.1097/00005537200207000-00005.

15. Al-Asaaf SM, Farhan MJ. Otitis externa in a localized area at the South of Jordan. Saudi Med J. 2000;21(10):928-30.

16. Nogueira JC, MeloDinizMde F, Lima EO, Lima ZN. Identification and antimicrobial susceptibility of acute external otitis microorganisms. Braz $J$ Otorhinolaryngol. 2008;74(4):526-30.

\section{How to cite this article:}

Shilpa K. Gokale, Anushka Devinkar, Drsuresh Sonth and Solabannavar, S.S. 2017. Bacteriological Study of Acute Otitis Externa in a Tertiary Care Hospital of a District in North Karnataka. Int.J.Curr.Microbiol.App.Sci. 6(9): 981-985. doi: https://doi.org/10.20546/ijcmas.2017.609.119 